\title{
Charge Transfer Properties in MEH-PPV/PS:MWCNTs Nanocomposites
}

\author{
Nadia Chehata $^{1 *}$, Olfa Dhibi ${ }^{1}$, Adnen Ltaief ${ }^{1}$, Ali Farzi ${ }^{2}$, Abdelaziz Bouazizi $^{1}$ \\ ${ }^{1}$ Equipe Composants Electroniques Organiques et Photovoltaïques Moléculaires, Département de Physique, Faculté des Sciences de \\ Monastir, Monastir, Tunisie; ${ }^{2}$ Department of Material and Polymer Engineering, Faculty of Engineering, Sabzevar Tarbiat Moallem \\ University, Sabzevar, Iran. \\ Email: * nadiachehata2@gmail.com
}

Received December $13^{\text {th }}, 2011$; revised January $15^{\text {th }}, 2012$; accepted January $28^{\text {th }}, 2012$

\begin{abstract}
The effect of composition and annealing temperature on charge transfer properties, in a donor/acceptor nanocomposites based on poly(2-methoxy-5-(2-ethyhexyl-oxy)-p-phenylenevinylene) (MEH-PPV) and MWCNTs functionalized with Polystyrene (PS:MWCNTs), have been investigated. The quenching of photoluminescence (PL) intensity of pure MEH-PPV, by adding different amounts of functionalized carbon nanotubes, exhibits that a photoinduced charge transfer has been occurred. Charge transfer efficiency was obtained for an acceptable concentration of PS:MWCNTs about $0.5 \mathrm{wt} \%$ and at annealed temperature of about $80^{\circ} \mathrm{C}$. Quenching efficiency studies imply that MEH-PPV/PS:MWCNTs nanocomposites reveal a high degree of PL quenching, reaching a value of $\eta=76.9 \%$.
\end{abstract}

Keywords: Poly(2-methoxy-5-(2-ethyhexyl-oxy)-p-phenylenevinylene) (MEH-PPV); Functionalized Carbon Nanotubes; Charge Transfer; Nanocomposites

\section{Introduction}

Carbon nanotubes (CNTs), reported by Lijima in 1991 [1], are long cylinders of covalently bonded carbon atoms. There are two basic types of CNTs: single-wall carbon nanotubes (SWNTs) and multi-wall carbon nanotubes (MWCNTs). SWNTs are considered as a single graphene sheet rolled into a cylinder and MWCNTs are consisted of several concentric cylindrical shells of graphene sheets. The particular structure of carbon nanotubes, their nanometer scale diameter, high flexibility, low density and high aspect ratio makes these nanofillers particularly attractive to be investigated as reinforcement in polymer nanocomposites and be integrated as active materials $[2,3]$. However, several issues concerning the dispersion of carbon nanotubes in the polymer matrices and its effects on the properties of the resulting nanocomposite polymers $[4,5]$ remain to be addressed. The functionalization of CNTs is an effective way to prevent the aggregation of carbon nanotubes and to improve their dispersion in solvents and within a polymer matrix $[6,7]$. The interfacial adhesion could be modified through interactions between the functional group on the nanotubes and the conjugated polymer for optimal nanocomposites properties, including optical properties based on charge transfer efficiency in devices addressed for photovoltaic

${ }^{*}$ Corresponding author. applications. It seems that functionalization of CNTs with polymers like polystyrene bring interesting behaviour to CNTs and improves its dispersion in polymer matrix. For this reason, we have considered in our research the possibility of investigating MWCNTs functionalized with Polystyrene (PS:MWCNTs) as acceptor nanofillers within MEH-PPV polymer. Based on optical measurements, this study contains two parts: 1) The effect of PS:MWCNTs concentrations on polymer nanocomposites; 2) The effect of thermal annealing treatment of MEH-PPV/PS:MWCNTs nanocomposites on charge transfer efficiency. The obtained results demonstrate that a photoinduced charge transfer has been occurred at MEH-PPV/PS:MWCNTs interfaces and exhibit a great effect of these parameters (nanofillers content and annealing treatment) on optical properties of MEH-PPV/ Carbon Nanotubes nanocomposites.

\section{Experimental}

MEH-PPV was purchased from Sigma Aldrich. The molecular weight of MEH-PPV was reported as $M_{n}=86,000$ $\mathrm{g} / \mathrm{mol}$. PS:MWCNTs were synthesized as following: 0.1 $\mathrm{g}$ of MWCNTs was suspended in $10 \mathrm{~g}$ of styrene and sonicated for 2 minutes, then this suspension was added into a water solution of surfactant (SDS), (0.1 g SDS in $90 \mathrm{~g}$ of distilled water) and sonicated for 2 minutes under 
stirring to create droplets. A stable mini-emulsion was obtained and it was transferred in a three necked round bottom flask connected with a condenser and gas inlet and outlet tubes. Oxygen was removed from mini-emulsion by means of a positive flow of nitrogen for $30 \mathrm{~min}$. Then, it was heated up to $70^{\circ} \mathrm{C}$ and little amount of ammonium persulphate (APS) was added as an initiator and the polymerization was carried out for 4 hours.

The nanocomposite films were prepared under ambient temperature by spin-coating from an homogenous chloroform dispersion of the blended materials onto glass suprasil. Blends of MEHPPV and PS:MWCNTs at weight ratios (PS:MWCNTs/MEH-PPV + PS:MWCNTs): from $0 \mathrm{wt} \%$ to $10 \mathrm{wt} \%$ were prepared by stirring for several hours in chloroform solvent at a viscosity of $10 \mathrm{mg} / \mathrm{ml}$. Individually dispersed PS:MWCNTs were apparent, while large aggregates were present in the solution containing MWCNTs without PS, even after a long period of ultrasonication. For thermal treatment study, MEH-PPV/ PS:MWCNTs $(0.5 \mathrm{wt} \%)$ samples were annealed under vacuum at various temperatures: $65^{\circ} \mathrm{C}, 80^{\circ} \mathrm{C}$ and $120^{\circ} \mathrm{C}$.

UV-visible absorption spectra were measured by PerkinElmer Instruments, Lambda 35 spectrophotometer. Photoluminescence spectra were collected with a JOBIN YVON-SPEX Spectrum One-CCD detector, cooled at liquid nitrogen temperature. The excitation wavelength was selected to be at $500 \mathrm{~nm}$.

\section{Results and Discussion}

This work will be based on investigating both absorption and photoluminescence spectra of nanocomposites as function of composition of PS:MWCNTs and annealing treatment. The obtained curves will be analyzed by exploiting two tools: the quenching efficiency parameter $(\eta)$ and the Stern-Volmer (SV) relation.

\subsection{Effect of PS:MWCNTs Concentration}

The absorption spectra of MEH-PPV/PS:MWCNTs nanocomposite films, for various concentrations of PS: MWCNTs, in the wavelength range from 400 to $700 \mathrm{~nm}$, are shown in Figure 1. Upon addition of PS:MWCNTs, the absorption peak of MEH-PPV (at $500 \mathrm{~nm}$ ) gradually decreases. It is probably due to the reduction of optical volume of MEH-PPV polymer [8]. Moreover, as concentration of PS:MWCNTs in MEH-PPV/PS:MWCNTs films increases up to $0.2 \mathrm{wt} \%$, the absorption edge shifts toward high wavelength wave further in the range of $\sim 5 \mathrm{~nm}$. Such red-shift of the absorption peak of MEH-PPV polymer is owing to the change of effective conjugation length of the conjugated polymer in the presence of carbon nanotubes [9]. In fact, the addition of CNTs to $\pi$-conjugated polymers was found to improve the quantum efficiency of $\pi$-conjugated polymers because the interaction between the highly delocalized $\pi$-electrons of CNTs and the $\pi$-electrons correlated with the lattice of the polymer skeleton. It is major to note that the UVvisible absorbance of nanocomposite films was almost completely dominated by MEH-PPV due to the very minor contributions of PS:MWCNTs. The quenching of the PL intensity of an appropriate donor polymer by a suitable acceptor material gives an indication of an effective photoinduced charge transfer at donor/acceptor interfaces $[10,11]$. Thus, the PL spectra of the MEH-PPV/PS: MWCNTs were investigated, as shown in Figure 2.

From Figure 2, it can be seen that the MEH-PPV polymer film shows strong photoluminescence between 525 and $750 \mathrm{~nm}$, with excitation at $500 \mathrm{~nm}$. The photoluminescence intensity is reduced when adding PS: MWCNTs nanofillers. The progressive decrease in PL intensities show that the excited fluorophore in the MEH-PPV backbone was quenched by the electronic interactions at the MEH-PPV/PS:MWCNTs nanoscale.

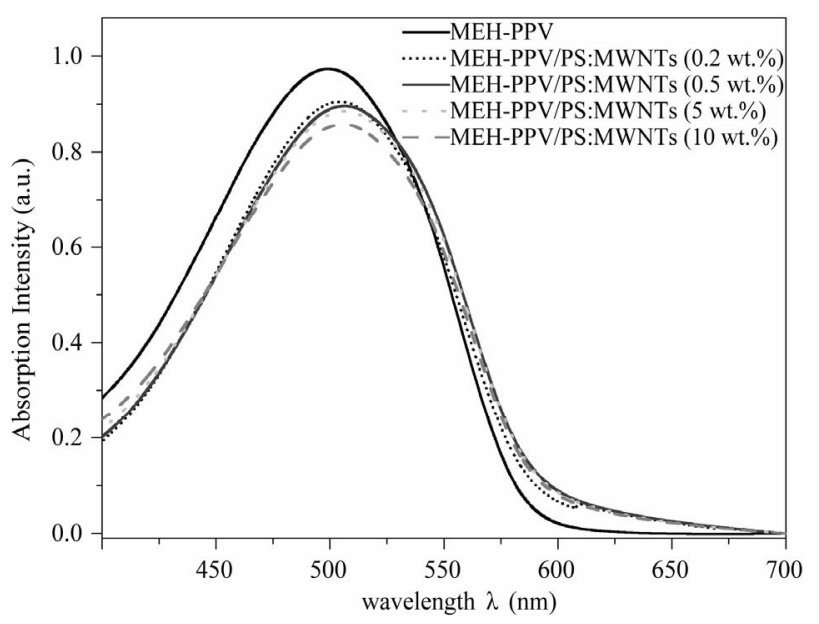

Figure 1. Absorption spectra of MEH-PPV/PS:MWCNTs nanocomposites with different concentrations of PS:MWCNTs.

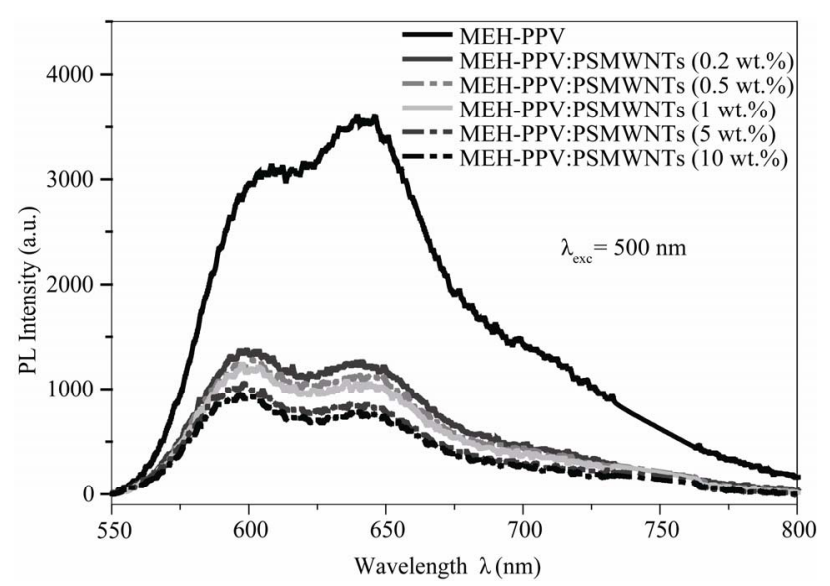

Figure 2. Photoluminescence spectra of MEH-PPV and MEHPPV/PS:MWCNTs nanocomposites. 
These results indicate that an efficient charge transfer has been occurred between MEH-PPV and PS:MWCNTs interfaces. Furthermore, the high length to diameter aspect ratio of MWCNTs greatly supports charge tunneling between the donor-acceptor systems through polystyrene (PS) shielding nanotubes. The efficient quenching of the PL emission shows that fast charge separation and slow charge recombination are consistently observed, thereby suggesting that functionalized MWCNTs (PS:MWCNTs) are expected to be a competitive candidate for the acceptor material in polymer organic solar cells.

The charge transfer process from the donor to the acceptor materials is occurred until the absorption of light and the dissociation of excitons, resulting on the separation of charges. Correlation between these processes is important to more understanding the charge transfer process. Thus, we introduce the quenching efficiency parameter $\eta_{C}$, expressed as [12]:

$$
\eta_{C}=1-\frac{\frac{I_{i C}}{A_{i C}}}{\frac{I_{o C}}{A_{o C}}}=1-\frac{I_{i C} A_{o C}}{A_{i C} I_{o C}}
$$

where, $A_{o c}$ : Absorbance intensity without PS:MWCNTs; $A_{i C}$ : Absorbance intensities with PS:MWCNTs; $I_{O C}$ : Integrated photoluminescence intensity without PS:MWCNTs; $I_{i C}$ : Integrated photoluminescence intensity with PS: MWCNTs.

The expression of quenching efficiency parameter $\eta_{C}$ link optical properties of donor-acceptor system at fundamental and excited states, which allows consequently to further understand the interaction between conjugated polymer and carbon nanofillers. Table 1 summarizes different parameters needed to calculate the quenching efficiency $\eta_{C}$ of elaborated samples. Figure 3 displays the variation of the quenching efficiency versus PS: MWCNTs concentrations. We can observe a rapid linear increase of quenching efficiency up to $0.5 \mathrm{wt} \%$, where the quenching efficiency $\eta_{\mathrm{C}}$ evolutes from $0 \%$ to $66.8 \%$.

Table 1. Quenching efficiency for different PS:MWCNTs concentrations in MEH-PPV/PS:MWCNTs nanocomposites.

\begin{tabular}{ccccc}
\hline PS:MWCNTs $(\mathrm{wt} \%)$ & $I_{i C}$ & $A_{i C}$ & $I_{i C} / A_{i C}$ & $\eta_{C}$ \\
\hline 0.0 & 398976.472 & 0.974 & 409626.770 & 0.000 \\
0.2 & 149164.638 & 0.905 & 164822.804 & 0.636 \\
0.5 & 136161.153 & 0.896 & 151965.573 & 0.668 \\
1.0 & 127715.278 & 0.896 & 142380.466 & 0.688 \\
5.0 & 104877.736 & 0.885 & 118505.916 & 0.744 \\
10.0 & & & & \\
& 94430.951 & 0.858 & 110059.383 & 0.769 \\
\hline
\end{tabular}

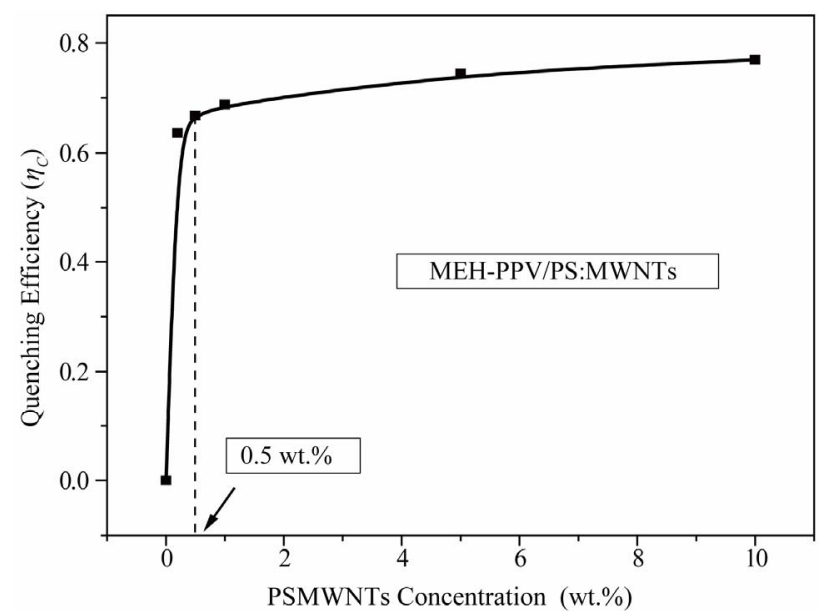

Figure 3. Variation of the quenching efficiency parameter as function of PS:MWCNTs concentration.

For higher concentrations, the quenching efficiency remains almost constant, passing from $66.8 \%$ to $76.9 \%$. We can interpret the obtained curve by means of the evolution of the interfacial area aspect with the increase of CNTs concentration. When the concentration of CNTs increases, they tend to agglomerate progressively, leading to inhomogeneous distribution of the nanotubes in the polymer matrix. When the concentration of PS: MWCNTs is greater than $0.5 \mathrm{wt} \%$, there is an ineffective dispersion of CNTs in MEH-PPV polymer. Thus, the quenching efficiency increases with less pronounced manner. The results indicate that a PS:MWCNTs concentration of $0.5 \mathrm{wt} \%$ is the optimum dispersion concentration for MEH-PPV/PS:MWCNTs nanocomposites. We can conclude that the ratio of electron acceptor material in donor polymer matrix is indispensible for photoinduiced charge transfer efficiency and it is crucial to determine the optimum concentration of each nanocomposite.

For further estimating the quenching process, the PL quenching characteristics of MEH-PPV/ PS:MWCNTs nanocomposites were analyzed using the Stern-Volmer $(S V)$ relation [13], which is the following:

$$
\frac{F_{o C}}{F_{C}}=1+K_{S V}^{C}[Q]
$$

where, $F_{o C}$ : The fluorescent intensity of pure MEH-PPV; $F_{C}$ : The fluorescent intensities with adding of PS: MWCNTs; $K_{S V}^{C}$ : The Stern-Volmer constant or quenching constant; $[Q]$ : The quencher concentration (PS: MWCNTs) in MEH-PPV/PS:MWCNTs nanocomposites. Generally, the SV relation is carried out to identify the nature of quenching mechanism, which falls into two processes [14]: 1) Static quenching through the formation of a ground state complex; and 2) Dynamic or collisional quenching due to diffusive collisions between the fluo- 
rescent and the quencher. Both types of quenching require an interaction between the quencher and fluorophore. In this study, the interaction between the MEHPPV (fluorescent) and CNTs (quencher) is studied by quencher concentration dependence on Stern-Volmer constant for different amounts of CNTs in polymer matrix. Figure 4 demonstrates the dependence of $F_{o C} / F_{C}$ fraction as a function of PS:MWCNTs quencher concentration $[Q]$. The obtained feature suggests that he quenching mechanism follows Stern-Volmer $(S V)$ equation. The $S V$ feature can be divided into two linear ranges: the first one with a slope of $K_{S V 1}^{C}=2.71$ for concentrations below $0.5 \mathrm{wt} \%$ and the second another with a slope of $K_{S V 2}^{C}=0.09$ for concentrations above $0.5 \mathrm{wt} \%$. As the value of Stern-Volmer constant decreases, the interaction between the quencher and fluorescent materials becomes less important. In our case, there is a great difference between the two quenching constants $\left(K_{S V 1}^{C}\right.$ and $\left.K_{S V 2}^{C}\right)$, which decrease significantly with the increase of PS: MWCNTs amounts. This result suggests that the interaction between MEH-PPV and PS:MWCNTs nanofillers for concentrations below $0.5 \mathrm{wt} \%$ (having the highest value of $K_{S V}^{C}$ ) is stronger than the one for nanofillers having concentrations above $0.5 \mathrm{wt} \%$. This result could be explained by two facts [15]: 1) Both MEH-PPV and PS:MWCNTs have a large $\pi$-conjugated system. The $\pi-\pi$ interaction between MEH-PPV and PS:MWCNTs may change the configuration of MEH-PPV, resulting in our case in a red-shift of absorption peak of MEH-PPV polymer (Figure 1); 2) The photoinduced charge transfer from excited MEH-PPV to PS:MWCNTs, for the concentrations less than $0.5 \mathrm{wt} \%$, was rapid. Early, we had demonstrated that the quenching efficiency (Figure 3) for concentrations less than $0.5 \mathrm{wt} \%$ increases with more pronounced manner. Moreover, the effective concentration of the quencher is dependent on the defects on its surfaces, which can affect the interfacial area aspect and the effective interaction of PS:MWCNTs with MEHPPV. To demonstrate the effect of defects, the concentration quencher can be modified to [12]:

$$
[Q]=\alpha C
$$

where $\alpha$ represents the ratio of the PS:MWCNTs surface area without defects to the total PS:MWCNTs one. The combination between Equation (2) and Equation (3) gives up the following equation:

$$
\frac{F_{o C}}{F_{C}}=1+K_{S V}^{C} \alpha C=1+K_{S V}^{\prime} C
$$

where the slope will be expressed as $K_{S V}^{\prime} C=K_{S V}^{C} \alpha$. Subsequently, the dependence of $F_{o C} / F_{C}$ fraction as a function of the quencher concentration exhibits that higher defect contents on the surfaces of PS:MWCNTs display lower slopes and so lower quenching constant. Thus, the defects related to great concentrations (more than $0.5 \mathrm{wt} \%$ ), will distort the MEH-PPV-PS:MWCNTs interaction and affects the donor-acceptor interfacial area aspect. This proves the obtained curve behavior of the variation of quenching efficiency parameter versus the quencher concentration. In conclusion, the photoinduiced charge transfer process is mostly depending on: the MEH-PPV/PS:MWCNTs interaction improvement, the interfacial area aspect and CNTs with less defects distribution.

\subsection{Effect of Annealing Temperature}

The effect of annealing temperature study is carried out at fixed concentration of PS:MWCNTs in MEH-PPV/PS: MWCNTs nanocomposite. The concentration is fixed at $0.5 \mathrm{wt} \%$, which represents the optimum concentration for the optimal charge transfer efficiency for MEH-PPV/ PS:MWCNTs nanocomposites.

The normalized UV-visible absorption spectra of the MEH-PPV/PS:MWCNTs nanocomposite films for different thermal annealing temperatures $\left(65^{\circ} \mathrm{C}, 80^{\circ} \mathrm{C}\right.$, $100^{\circ} \mathrm{C}$ and $120^{\circ} \mathrm{C}$ ) are shown in Figure 5. As the annealing temperature increases, the absorption spectra are slightly red-shifted of about $3 \mathrm{~nm}$, indicating a shift in the scale of energy about $413 \mathrm{eV}$. The thermal annealing contributes to the increase of $\pi$-electron delocalization and optical $\pi-\pi^{*}$ transition, which leads to an enhancement of interchain interaction inside the MEH-PPV chains and a decrease of the band-gap between $\pi$ and $\pi^{*}$ molecular orbitals $[15,16]$. Moreover, as the annealing temperature increases, the absorption spectra are notably broadened in the short energy region. The broadened of the long wavelength absorption of the nanocomposite films is attributed to the tendency of PS:MWCNTs to

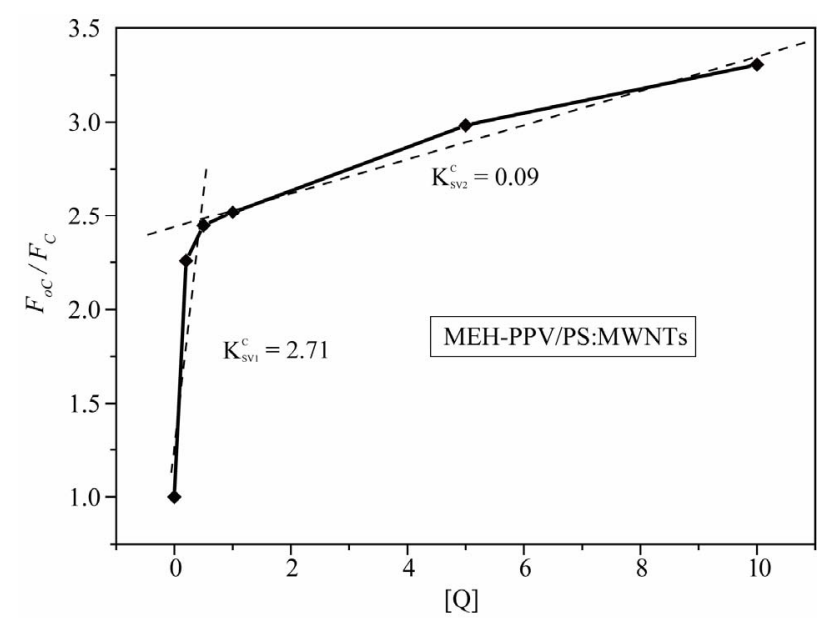

Figure 4. Variation of $F_{o C} / F_{C}$ fraction as function of PS: MWCNTs concentration within polymer matrix. 
form aggregation [17]. Besides, the annealing temperature dependence on PL spectra between 525 and $800 \mathrm{~nm}$, with excitation at $500 \mathrm{~nm}$, isobserved. Figure 6(a) displays the PL spectra of un-annealed and annealed films under various temperatures of $65^{\circ} \mathrm{C}, 80^{\circ} \mathrm{C}, 100^{\circ} \mathrm{C}$ and $120^{\circ} \mathrm{C}$. We can observe that as the annealing temperature increases, the PL intensity is considerably decreased. For un-annealed and annealed nanocomposite films at $120^{\circ} \mathrm{C}$, the ratio of relative intensity of the main peak is about 0.64 .

Thus, the decrease of PL intensity indicates that the thermal treatment is a favorable agent to reduce the charges recombination, improve the dissociation of excitons, and then improve the charge transfer in MEHPPV/PS:MWCNTs nanocomposite films. In fact, the thermal annealing process allows a rearrangement of the polymer chains and an orientation of nanotubes within the polymer matrix, leading to an increase of interfaces between MEH-PPV and MWCNTs for optimal charge dissociation efficiency. To investigate the effect of annealing treatment on MEH-PPV/PS:MWCNTs nanofillers nanoscale interactions, the PL data are normalized relative to their maximum emission, as shown in Figure 6(b). We notice that annealing temperature dramatically influence on the normalized PL spectra. For the un-annealed MEH-PPV/PS:MWCNTs nanocomposite film, a PL spectrum with a main peak at $588 \mathrm{~nm}$ and a shoulder at $624 \mathrm{~nm}$, is observed. The increase of annealing temperature, significantly, changes on the shape of the overall PL spectra: a reduction of intensity of the main peak, an increase in intensity of shoulder peak, and a red-shift of the whole PL spectrum. For annealed films at $80^{\circ} \mathrm{C}$ and $120^{\circ} \mathrm{C}$, an intensity peak appears at 594 and $642 \mathrm{~nm}$, respectively.

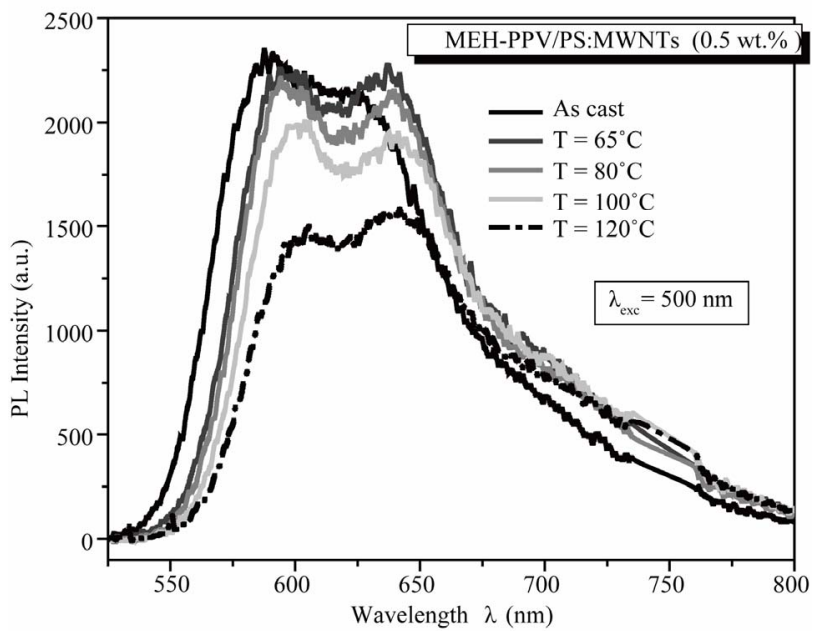

(a)
As we have previously revealed, annealing temperature is an important factor influencing the optical properties of MEH-PPV/PS:MWCNTs nanocomposites by improving the photoinduiced charge dissociation. As function of temperature, the quenching efficiency parameter $\eta_{T}$ will be introduced, in order to more emphasize the effect of annealing temperature. The quenching efficiency parameter $\eta_{T}$, at various annealing temperatures, is expressed as following:

$$
\eta_{T}=1-\frac{\frac{I_{i T}}{A_{i T}}}{\frac{I_{o T}}{A_{o T}}}=1-\frac{I_{i T} A_{o T}}{A_{i T} I_{o T}}
$$

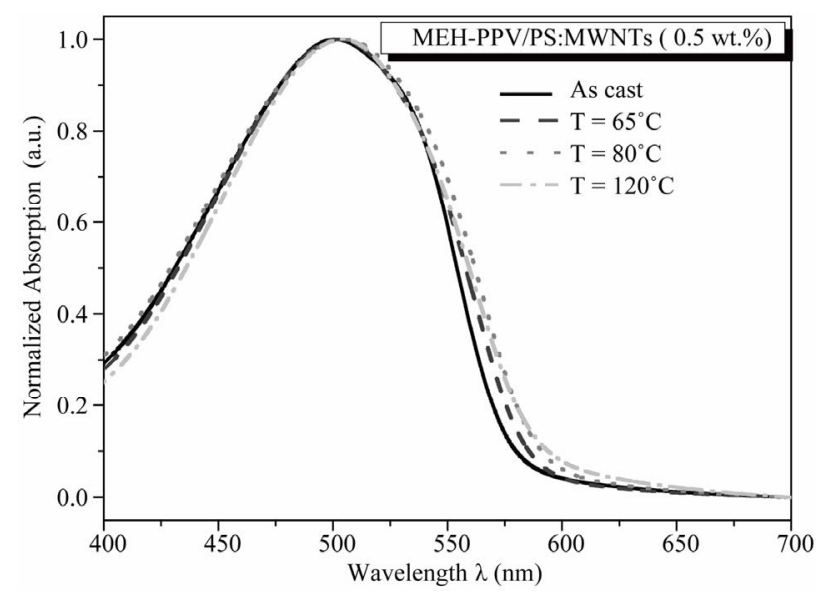

Figure 5. Normalized optical absorption spectra of the ascast nanocomposite film and the nanocomposite films after annealing temperatures, with MEH-PPV/PS:MWCNTs (0.5 wt\%).

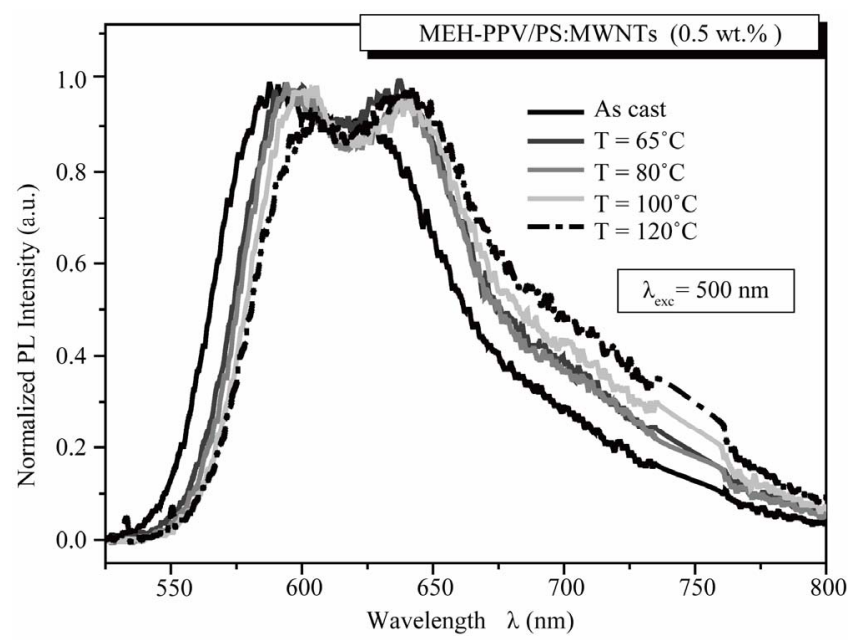

(b)

Figure 6. (a) PL spectra of un-annealed and annealed nanocomposite films, for MEH-PPV/PS:MWCNTs (0.5 wt\%) nanocomposites; (b) Normalized PL spectra of un-annealed and annealed nanocomposite films for MEH-PPV/PS:MWCNTs (0.5 wt\%) nanocomposites. 
where, $A_{o T}$ : The absorbance intensity for un-annealed nanocomposite; $A_{i T}$ : The absorbance intensities for annealed nanocomposites at different annealing temperatures; $I_{O T}$ : Integrated photoluminescence intensity for unannealed nanocomposites; $I_{i T}$ : Integrated photoluminescence intensity for annealed nanocomposites at different annealing temperatures.

These parameters are summarized in Table 2 and used to follow the quenching efficiency variation as a function of thermal annealing temperatures, as shown in Figure 7. The quenching efficiency is almost increased linearly until an annealing temperature of $80^{\circ} \mathrm{C}$. When the annealing temperature is risen from $80^{\circ} \mathrm{C}$ to $120^{\circ} \mathrm{C}$, the quenching efficiency is dramatically dropped. The reduction in quenching efficiency at high annealing temperature can be explained by various factors: 1) It can be seen from Table 2 that absorbance intensity shows a decrease at high temperatures, which is related to the decrease on the thickness of photoactive layer after thermal processing [18]. This can probably contributed to reduce the number of photons absorbed in the photoactive layer as well as the ratio of generated excitons; 2) The reduction of $\eta_{T}$ may be related to phase segregation, which is related to the absence of privilegious orientation of carbon nanotubes under annealing temperature.

The intensity of fluorescence was quenched significantly since annealing temperature is progressively applied. In order to distinguish between dynamic or static quenching process and to identify the basic mechanism, the Stern-Volmer plot of the dependence on annealing temperature of nanocomposite films was carried out (Figure 8).

The Stern-Volmer relation for different annealing temperatures is:

$$
\frac{F_{o T}}{F_{T}}=1+K_{S V}^{T}[T]
$$

where, $F_{o T}$ : PL intensity of un-annealed MEH-PPV/PS: MWCNTs nanocomposite; $F_{T}$ : PL intensities of annealed MEH-PPV/PS:MWCNTs nanocomposites; $K_{S V}^{T}$ : SternVolmer constant; $[T]$ : Applied annealing temperature.

Table 2. Quenching efficiency parameter for different annealing temperatures in MEH-PPV/PS:MWCNTs nanocomposites.

\begin{tabular}{ccccc}
\hline $\mathrm{T}\left({ }^{\circ} \mathrm{C}\right)$ & $\left(I_{i T}\right)$ & $\left(A_{i T}\right)$ & $I_{i T} / A_{i T}$ & $\left(\eta_{T}\right)$ \\
\hline As cast & 266879.575 & 0.574 & 464866.008 & 0.000 \\
65 & 276874.416 & 0.707 & 391618.693 & 0.158 \\
80 & 261745.794 & 0.826 & 316883.528 & 0.318 \\
100 & 246540.177 & 0.655 & 376397.217 & 0.190 \\
120 & 203804.506 & 0.480 & 424592.721 & 0.087 \\
\hline
\end{tabular}

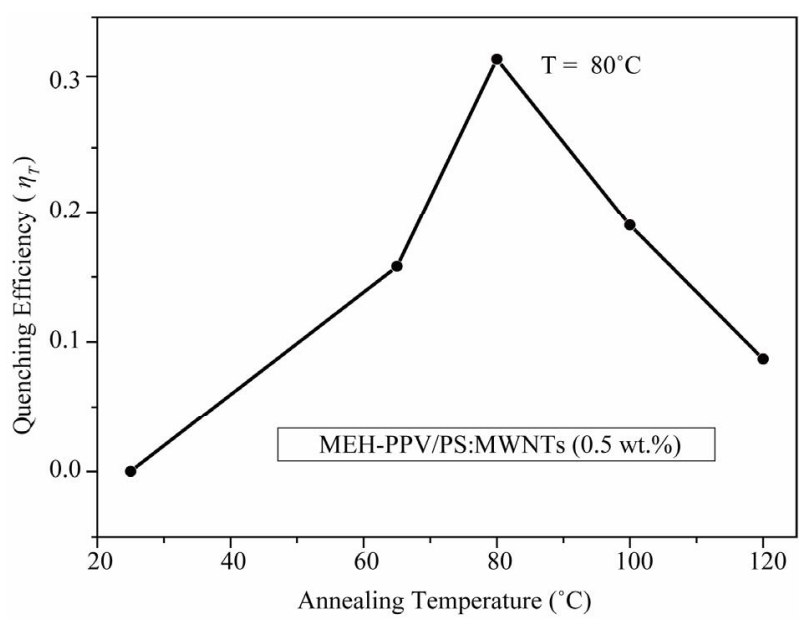

Figure 7. Variation of quenching efficiency versus the annealing temperatures of organic nanocomposites.

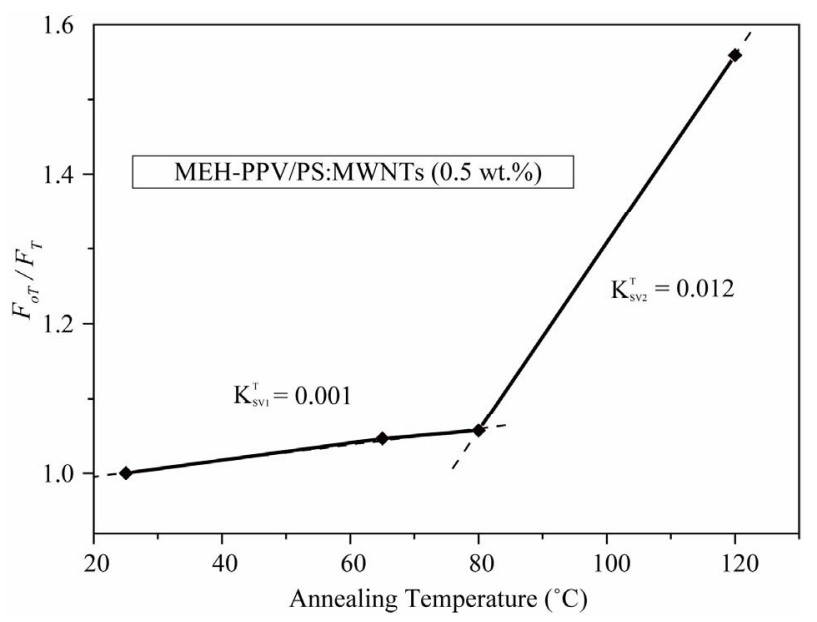

Figure 8. Stern-volmer plot for the MEH-PPV/PS:MWCNTs nanocomposites, for different annealing temperatures.

The annealing temperature dependence on SternVolmer constant, shown in Figure 8, is presented by two linear behaviors with transition temperature of $80^{\circ} \mathrm{C}$. The Stern-Volmer constant $K_{S V}^{T}$ is enhanced with increasing annealing temperature. Wang et al. [19] reported that dynamic quenching is expected to be more effective at elevated temperature where $K_{S V}^{T}$ will increase due to increased rate of diffusion of the quencher within the fluorophore, whereas static quenching will became less effective at elevated temperature due to decreased stability of the complex and thereby reducing $K_{S V}^{T}$. In this case, the increase of Stern-Volmer constant $K_{S V}^{T}$ indicates that dynamic quenching is responsible for the decrease in emission intensity of MEH-PPV polymer upon addition of PS:MWCNTs. It is important to note that the collision between the quencher and the fluorophore affects only the excited state of the fluorophore; no changes in the absorption spectra are expected. In contrast, the formation of a ground state complex in static quenching will 
disturb the absorption spectra of the fluorophore upon adding of the quencher. As shown in Figure 5, the absorption spectra indicate a red spectral shift. In this context, the result supports the interpretation of Zheng et al. $[15,16]$, suggesting that a photoinduiced charge transfer complex could be formed in excited state rather than in the ground state. Thus, in our case, both quenching mechanisms can be considered to arise from complex formation.

\section{Conclusion}

To conclude, we have elaborated and optically characterized a donor/acceptor system holding MEH-PPV/PS: MWCNTs nanocomposites, by reporting the effect of composition and annealing temperature. Adding PS: MWCNTs into MEH-PPV induces great quenching of the photoluminescence of the MEH-PPV, indicating a strong charge transfer from the MEH-PPV to PS:MWCNTs. Quenching efficiency studies imply that MEH-PPV/PS: MWCNTs nanocomposite exhibited a higher degree of PL quenching $(\eta=76.9 \%)$. Moreover, it was revealed that photoinduced transfer is greatly affected by the dispersion of the carbon nanofillers. We found that, for these nanocomposites, the optimum dispersion of PS: MWCNTs amounts was $0.5 \mathrm{wt} \%$. The effect of annealing temperature for MEH-PPV/PS:MWCNTs nanocomposites, for the optimum concentration, was further studied. We have noticed that as the annealing temperature increases, the PL intensity is considerably decreased. Thus, the thermal treatment is a favorable agent to improve the dissociation of excitons and then improve the charge transfer in MEH-PPV/PS:MWCNTs nanocomposites. The quenching efficiency and Stern-Volmer relations exhibit that $80^{\circ} \mathrm{C}$ is the optimum annealing temperature. The present results indicated that the MEH-PPV/PS: MWCNTs formed an effective interpenetrating network in the blend films, which favors exciton dissociation in the interfacial area and charge transfer in the corresponding domains. The profound understanding of charge transfer behavior tunes a key step on the performance of these nanocomposites to be used as photoactive materials in organic electronics such as photovoltaic devices.

\section{Acknowledgements}

N. Chehata gratefully acknowledges Mr. Joel DAVENAS to the reception in Laboratory of Polymer Materials and Biomaterials, University Claude Bernard, Lyon 1, to realize this experimental work.

\section{REFERENCES}

[1] S. Iijima, "Helical Microtubules of Graphitic Carbon," Nature, Vol. 354, No. 6348, 1991, pp. 56-58. doi: $10.1038 / 354056 \mathrm{a} 0$
[2] P. Fournet, J. N. Coleman, B. Lahr, A. Drury, W. J. Blau, D. F. O'Brien and H.-H. Hörhold, "Enhanced Brightness in Organic Light-Emitting Diodes Using a Carbon Nanotube Composite as an Electron-Transport Layer," Journal of Applied Physics, Vol. 90, No. 2, 2001, pp. 969-975. doi:10.1063/1.1383023

[3] E. Kymakis and G. A. J Amaratunga, "Single-Wall Carbon Nanotube/Conjugated Polymer Photovoltaic Devices," Applied Physics Letters, Vol. 80, No. 1, 2001, pp. 112114. doi:10.1063/1.1428416

[4] M. Moniruzzaman and K. I. Winey, "Polymer Nanocomposites Containing Carbon Nanotubes," Macromolecules, Vol. 39, No. 16, 2006, pp. 5194-5205. doi:10.1021/ma060733p

[5] F. Hussain, M. Hojjati, M. Okamoto and R. E. Gorga, "Polymer-Matrix Nanocomposites, Processing, Manufacturing, and Application: An Overview," Journal of Composite Materials, Vol. 40, No. 17, 2006, pp. 1511-1575. doi:10.1177/0021998306067321

[6] N. G. Sahooa, S. Ranab, J. W. Chob, L. Li and S. H. Chana, "Polymer Nanocomposites Based on Functionalized Carbon Nanotubes," Progress in Polymer Science, Vol. 35, No. 7, 2010, pp. 837-867. doi:10.1016/j.progpolymsci.2010.03.002

[7] D. E. Hill, Y. Lin, A. M. Rao, L. F. Allard and Y. P. Sun, "Functionalization of Carbon Nanotubes with Polystyrene," Macromolecules, Vol. 35, No. 25, 2002, pp. 94669467. doi: $10.1021 / \mathrm{ma} 020855 \mathrm{r}$

[8] A. Ltaief, A. Bouazizi, J. Davenas, R. B. Chaâbane and H. B. Ouada, "Electrical and Optical Properties of Thin Films Based on MEH-PPV/Fullerene Blends," Synthetic Metals, Vol. 147, No. 1-3, 2004, pp. 261-266. doi:10.1016/i.synthmet.2004.09.018

[9] V. Lordi and N. Yao, "Molecular Mechanics of Binding in Carbon Nanotubes-Polymer Composites," Journal of Materials Research, Vol. 15, No. 12, 2000, pp. 27702779. doi:10.1557/JMR.2000.0396

[10] A. Ltaief, A. Bouazizi, J. Davenas and P. Alcouffe, "Influence of the Processing Conditions on Charge Transfer and Transport Properties in Poly(2-methoxy-5-(2'-ethylhexyloxy) 1-4-phenylenevinylene): $\mathrm{C}_{60}$ Composites for Photovoltaics," Thin Solid Films, Vol. 516, No. 7, 2008, pp. 1578-1583. doi:10.1016/j.tsf.2007.07.191

[11] N. S. Sariciftci, L. Smilowitz, A. J. Heeger and F. Wudl, "Photoinduced Electron Transfer from a Conducting Polymer to Buckminsterfullerene," Science, Vol. 258, No. 5087, 1992, pp. 1474-1476. doi:10.1126/science.258.5087.1474

[12] Q. Liu, Z. Liu, X. Zhang, L. Yang, N. Zhang, G. Pan, S. Yin, Y. Chen and J. Wei, "Polymer Photovoltaic Cells Based on Solution-Processable Graphene and P3HT," Advanced Functional Materials, Vol. 19, No. 6, 2009, pp. 894-904. doi:10.1002/adfm.200800954

[13] B. Valeur, "Molecular Fluorescence: Principals and Applications," Wiley-VCH, Weinheim, 2002, pp. 72-124.

[14] J. R. Lakowicz, "Principles of Fluorescence Spectroscopy," Plenum Press, New York, 1983.

[15] M. Zheng, F. Bai, F. Li, Y. Li and D. Zhu, "The Interaction between Conjugated Polymer and Fullerenes," Jour- 
nal of Applied Polymer Science, Vol. 70, No. 3, 1998, pp. 599-603.

doi:10.1002/(SICI)1097-4628(19981017)70:3<599 ::AID -APP22>3.0.CO;2-V

[16] G. D. Sharma, V. S. Choudhary and M. S. Roy, "Effect of Annealing on the Optical, Electrical, and Photovoltaic Properties of Bulk Hetero-Junction Device Based on PPAT: TY Blend," Solar Energy Materials and Solar Cells, Vol. 91, No. 4, 2007, pp. 275-284. doi:10.1016/j.solmat.2006.09.006

[17] H. Jin, Y. Hou, F. Teng, P. Kopola , M. Tuomikoski and A. Maaninen, "Effect of Molecular Aggregation by Thermal Treatment on Photovoltaic Properties of MEH-PPV: Fullerene-Based Solar Cells," Solar Energy Materials and Solar Cells, Vol. 93, No. 2, 2009, pp. 289-294. doi:10.1016/j.solmat.2008.10.025

[18] W. Lee and O. O. Park, "The Effect of Different Heat Treatments on the Luminescence Efficiency of Polymer Light-Emitting Diodes," Advanced Materials, Vol. 12, No. 11, 2000, pp. 801-804.

doi:10.1002/(SICI)1521-4095(200006)12:11<801: :AIDADMA801>3.3.CO;2-7

[19] J. Wang, D. Wang, D. Moses and A. J. Heeger, "Dynamic Quenching of 5-(2'-Ethyl-Hexyloxy)-P-Phenylene Vinylene (MEH-PPV) by Charge Transfer to a $\mathrm{C}_{60}$ Derivative in Solution," Journal of Applied Polymer Science, Vol. 82, No. 10, 2001, pp. 2553-2557. doi:10.1002/app.2106 